\title{
Status of selected ion flow tube mass spectrometry; accomplishments and challenges in breath analysis and other areas.
}

\section{David Smith $^{\dagger 1}$ and Patrik Španěl ${ }^{1,2}$}

${ }^{1}$ Institute for Science and Technology in Medicine, School of Medicine, Keele University, Thornburrow Drive, Hartshill, Stoke-on-Trent ST4 7QB, UK

${ }^{2}$ J. Heyrovsky Institute of Physical Chemistry of Science, Academy of Science of the Czech Republic, Dolejškova 3, 18223 Prague 8, Czech Republic

$10 \dagger$ Author for correspondence:

Tel.: +441782674988

E-maild.smith@keele.ac.uk

\section{Acknowledgements}

We acknowledge funding by The Czech Science Foundation GACR project 13-28882S.

\section{ABSTRACT}

This "Perspective" reflects our observations of recent accomplishments made using SIFTMS. Only brief descriptions are given of SIFT-MS as an analytical method and of the recent extensions to the underpinning analytical ion chemistry required to realise more robust analyses. The challenge of breath analysis is given special attention because, when achieved, it renders analysis of other air media relatively straightforward. Brief overviews are given of recent SIFT-MS breath analyses by leading research groups, noting the desirability of detection and quantification of single volatile biomarkers rather than reliance on statistical analyses, for breath analysis is to be accepted into clinical practice. A "SWOT" analysis of SIFT-MS is made which should increase its utility for trace gas analysis.

Keywords: selected ion flow tube mass spectrometry, SIFT-MS, breath analysis, metabolites, volatile organic compounds, small molecules, real time analysis, trace gas analysis, cystic fibrosis, cancer, SWOT analysis. 
Practitioners of selected ion flow tube mass spectrometry, SIFT-MS, have already eloquently described its unique strengths amongst the techniques available to gas phase analysis. So, in this "Perspectives" article we do not again describe the SIFT-MS technique in detail, but rather we simply briefly outline the physics and ion chemistry that underpin the technique in order to facilitate later criticisms, outline the major successes that differentiate it from other gas phase analytical techniques and then reveal the weaknesses of the technique and its shortcomings in analysis. An important aspect of this article will be to comment critically on recent endeavours to utilize SIFT-MS via its different modes of operation and to summarise results of some recent studies. We attempt to suggest more profitable directions for its future exploitation, especially when related to clinical diagnosis by breath analysis of specific diseases for which SIFT-MS has a special and possibly vital role to play. In this regard, direct breath analysis and the detection and quantification of volatile disease "biomarkers" is of special interest for reasons that were touched upon in our recent short perspective [1] and will be expanded in the present article using topical examples of the work from different research groups. We will also reveal our own in-train developments of the instrumentation that are designed to increase versatility and value for trace gas analysis of humid samples of air, exhaled breath and the headspace of biogenic fluids such as bacterial cell cultures, which we anticipate will increase its appeal and attraction to researchers in other topics in which real time trace gas analysis has an important contribution to make.

\section{Selected ion flow tube mass spectrometry, SIFT-MS}

\section{- Overview}

The principle and operation of SIFT-MS has been given in several reviews [2-5]. It is sufficient to say here that it relies on the chemical ionization by selected reagent ions, $\mathrm{H}_{3} \mathrm{O}^{+}$, $\mathrm{NO}^{+}$and $\mathrm{O}_{2}^{+\cdot}$, of trace analyte compounds in air/breath samples introduced at a controlled flow rate into a helium-buffered flow tube. Reactions occur between the selected reagent ion and the composite analyte molecules that produce characteristic product (analyte) ions that are identified and their currents (count rates) measured by a downstream analytical mass spectrometer/detection system. The identified analyte ions are related to specific neutral trace compounds in the air/breath from an in-depth knowledge of the ion-molecule chemistry and their count rates, coupled with physical parameters such as carrier (helium) gas and sample gas flow rates, and are converted to concentrations of the neutral trace analyte molecules in the sample[6]. A proper appreciation of the complex ion chemistry occurring in SIFT-MS reactors, especially when humid samples are being analysed, is critical to achieving accurate trace gas analysis and we comment on some perceived short comings in this aspect later in this article.

Two acquisition modes of reagent ion and analyte ion count rates are used, viz. the full scan mode, FSM, in which all ions within a chosen mass-to-charge ratio, $\mathrm{m} / \mathrm{z}$, are sampled and integrated over several scans, and the multiple ion monitoring mode, MIM, in which all the reagent ions and a few analyte ions are sampled and counted by rapidly switching the analytical mass spectrometer. Using MIM, greater precision of measurement can be realized and changing concentration time profiles of several trace metabolites in single breath exhalation to be obtained simultaneously $[\underline{6}, \underline{7}]$. Currently, SIFT-MS instrumentation can achieve reliable quantification down to one part-per-billion by volume, ppbv, in one second 
of analyte ion integration $[\underline{8}, \underline{9}]$. Even sub-ppbv sensitivity has been claimed $[\underline{10}, \underline{11}$, typically using integration times in the order of minutes. Analyses of various media have been carried out using SIFT-MS by us and others [12-18], not least of exhaled breath [19-24], the results of which have been summarized in many research papers and some authoritative reviews [2-4, 25-29]. We later critically appraise some of the more recently reported studies.

The extension of the SIFT-MS data acquisition approach was made possible by software developments that allow the count rates of all reagent and analyte ions to be determined at integer $m / z$ values across the full mass spectral scan range available using the MIM approach. The $\mathrm{m} / \mathrm{z}$ range accessible in the current Profile 3 instruments is up to 300 . This allows statistical assessment of the mass spectral data and thus a careful critique of the use and misuse of the PCA approach to the identification of diagnostic biomarkers.

\section{- Ion chemistry}

As has been pointed out, SIFT-MS analysis depends on chemical ionisation using (precursor) reagent ions, $\mathrm{H}_{3} \mathrm{O}^{+}, \mathrm{NO}^{+}$or $\mathrm{O}_{2}^{+*}$, to generate the characteristic product (analyte) ions of the trace molecules in the sample being analysed [4]. The resulting analytical spectra are much simpler and more easily interpreted than those obtained using electron ionisation to analyse complex media like exhaled breath. Thus, $\mathrm{m} / \mathrm{z}$ overlaps of analyte ions are fewer allowing trace compounds in air/breath samples to be unambiguously analysed more rapidly than when using pre-concentration and separation techniques in association with gas chromatography/mass spectrometry (GC-MS). This precious feature of SIFT-MS facilitates real-time trace gas analysis, including the analysis of single breath exhalations in real time, as discussed later.

However, there is a sting in the tail! It is essential that the complex ion chemistry taking place in the flow tube that is the ion chemistry reactor be thoroughly understood and proper ionmolecule kinetics data obtained to achieve accurate trace gas analyses. Such knowledge is not easily acquired; it has needed decades of research work by the authors to have gained sufficient understanding to conceive of and implement SIFT-MS as the reliable analytical technique it has become. Even under the truly thermalized conditions of SIFT-MS, the ion chemistry can be rich and several reaction mechanisms may occur that have to be understood in order to interpret the analytical mass spectra. Complications are minimised by the use of helium carrier gas, because it is relatively inert chemically, but even helium atoms are intimately involved in some of the ionic reactions, especially those involving the formation of adduct ions, as mentioned below.

The reactions of the three available reagent ions are very different and each must be understood if these reactions are to be used for analysis. $\mathrm{H}_{3} \mathrm{O}^{+}$ions react with most molecules, $\mathrm{M}$, including volatile organic compounds (VOCs), by the process of proton transfer resulting in $\mathrm{MH}^{+}$ions, but it is important to realise that for many organic molecules spontaneous dissociation of the nascent $\left(\mathrm{MH}^{+}\right)^{*}$ ions can occur, often with the ejection of an $\mathrm{H}_{2} \mathrm{O}$ molecule. Obviously, if this is not appreciated then gas analysis will be inaccurate and even meaningless. The analogous ion chemistry underpinning the PTR-MS drift tube is more complicated [8], given that both the reagent and analyte ions are suprathermal (kinetically excited) promoting break-up of the analyte ions in collisions with heavy nitrogen and oxygen carrier gas molecules rather than lighter helium carrier gas atoms used in SIFT-MS. We discuss this phenomenon later in relation to our recent development of our SIFDT-MS. $\mathrm{NO}^{+}$ ions react with VOCs by several defined mechanisms, including charge transfer producing $\mathrm{M}^{+}$ions, hydride ion transfer producing $(\mathrm{M}-\mathrm{H})^{+}$ions and by ion-molecule association 
producing $\mathrm{NO}^{+} \mathrm{M}$ adduct ions. $\mathrm{O}_{2}^{+\cdot}$ ions react by charge transfer with most molecules, which with small molecules like $\mathrm{NH}_{3}$ and $\mathrm{NO}$ is non-dissociative producing the parent radical cation (e.g. $\mathrm{NH}_{3}{ }^{+}$and $\mathrm{NO}^{+}$). However, $\mathrm{O}_{2}{ }^{+\bullet}$ reacts with most VOCs producing two or more fragment ions, and this minimises its value as a SIFT-MS reagent ion. This critical ion chemistry knowledge has been acquired by many studies of the reactions of these three reagent ions with groups and homologous series of many biogenic VOCs, [5, 30-32] in order to build the kinetics library needed for SIFT-MS analyses. [6, 9$]$

Detailed discussion of the relevant ion chemistry is given in the cited references. But it is vital now to discuss two aspects of it that are often not appreciated by those who attempt to advance SIFT-MS by increasing analytical sensitivity and to extend its application. These related to the analysis of very humid samples such as exhaled breath and the use of carrier gas other than inert helium, especially nitrogen. Inevitably, especially when exploiting $\mathrm{H}_{3} \mathrm{O}^{+}$ and $\mathrm{NO}^{+}$reagent ions for analysis, hydrated ions of both the reagent ion efficiently form, viz. $\mathrm{H}_{3} \mathrm{O}^{+}\left(\mathrm{H}_{2} \mathrm{O}\right)_{1,2,3}$ and $\mathrm{NO}^{+}\left(\mathrm{H}_{2} \mathrm{O}\right)_{1,2}$, and the analyte ions, viz. $\mathrm{MH}^{+}\left(\mathrm{H}_{2} \mathrm{O}\right)_{1,2,3}$ and $(\mathrm{M}-$ $\mathrm{H})^{+}\left(\mathrm{H}_{2} \mathrm{O}\right)_{1,2}$. The hydrated reagent ions must be considered as additional reagent ions in the analysis and this is not a trivial task, because these cluster ions are continuously formed along the length of the flow tube and so the reaction times of these additional reagent ions have to be estimated. We have addressed these complicating aspects of SIFT-MS analysis in detail, and constructed appropriate analytical expressions that involve the rates of these hydration processes $[\underline{6}, \underline{32}, \underline{33}]$. This would not have been possible without a detailed understanding of the ion hydration process we have acquired by many experimental investigations [30, 31]. Thus, trace gas analysis can now be achieved to acceptable accuracy in very humid samples, as we have demonstrated in several papers $[\underline{9}, \underline{18}, \underline{28}, \underline{34}, \underline{35}]$.

A closely related phenomenon is the formation of weakly-bound adducts by the association of the reagent ions with the other abundant molecules of the sample gas i.e. $\mathrm{N}_{2}, \mathrm{O}_{2}$ and, in exhaled breath, $\mathrm{CO}_{2}$. Thus, adduct ions such as $\mathrm{H}_{3} \mathrm{O}^{+} \mathrm{N}_{2}, \mathrm{H}_{3} \mathrm{O}^{+} \mathrm{CO}_{2}, \mathrm{NO}^{+} \mathrm{CO}_{2}, \mathrm{O}_{2}{ }^{+\cdot} \mathrm{O}_{2}$ and $\mathrm{O}_{2}{ }^{+} \mathrm{CO}_{2}$ can clearly be detected in a mixture of the helium carrier gas and dry air. The danger is that these adduct ions may be isobaric with an analyte ion formed in the analysis of some common trace compounds in biogenic mixtures and then quantification can be compromised. For example, $\mathrm{H}_{3} \mathrm{O}^{+} \mathrm{N}_{2}$ ions are isobaric with protonated ethanol $(\mathrm{m} / \mathrm{z} 47)$ and $\mathrm{H}_{3} \mathrm{O}^{+} \mathrm{CO}_{2}$ is isobaric with the monohydrate of protonated acetaldehyde $(\mathrm{m} / z$ 63). Whilst these two adduct ion species are very reactive, especially with $\mathrm{H}_{2} \mathrm{O}$ molecules, they are formed in the analysis of humid exhaled breath and must be accounted for to achieve accurate analyses. [36]. Similarly, the formation of $\mathrm{O}_{2}{ }^{+\bullet} \mathrm{CO}_{2}(\mathrm{~m} / \mathrm{z} 76)$ when using $\mathrm{O}_{2}{ }^{+\bullet}$ reagent ions to analyse $\mathrm{CS}_{2}$ in exhaled breath is a real problem (analyte ion in this case is $\mathrm{CS}_{2}{ }^{+}$) [37]. The formation of $\mathrm{H}_{3} \mathrm{O}^{+} \mathrm{N}_{2}$ will be particularly efficient when using nitrogen carrier gas that has been used in recent SIFT-MS analyses [38] or air as it is routinely used in PTR-MS [39, 40]. An essential point to make is that even though these adduct ions are not immediately detected by the downstream analytical mass spectrometer system, they are surely formed and undergo reactive loss especially with $\mathrm{H}_{2} \mathrm{O}$ molecules and other VOCs. These complications are generally not understood by most SIFT-MS and PTR-MS users and so for accurate analyses they must rely on calibration of their instrument using standard mixtures. But there are even dangers in this approach unless the calibrations are carried out at calibrant gas concentrations that are close to the concentrations in the sample mixtures to be analysed. It is our contention that over reliance on such calibrations without a good understanding of the analytical ion chemistry can lead to unrecognised inaccuracies. This is especially so when wide variations in trace gas (metabolite) concentrations occur in humid air/breath samples and when opportunistic variations in reactor conditions such as sample flow rate and variable $E / N$ in PTR-MS are exploited ostensibly to improve analyses. 
Finally, it is important to recognise that all users of SIFT-MS will not have a thorough background in ion chemistry and cannot be expected to appreciate all these nuances. However, to introduce a new compound into the analytical kinetics library requires an appreciation of this ion chemistry and thus will usually need the input of experts in the field.

\section{- Breath analysis}

SIFT-MS is now used to analyse air samples for trace VOCs in several areas of research, as listed in our recent reviews [2-4, 25-29]. However, it is in breath analysis where the real analytical challenges are revealed, because exhaled breath is supersaturated with water vapour, which most analytical techniques are not able to cope with when de-humidification is often required that can partially, which can partially or totally remove some trace compounds from the breath sample. But SIFT-MS not only copes with the high humidity but actually measures the humidity providing a very valuable method to check sample humidity (water vapour content), which for exhaled breath is always close to $6 \%$ by volume, and of sampling integrity $[\underline{9}, \underline{41}]$. Thus, breath analysis is directed towards identifying abnormal concentrations of VOC metabolites, distinguishing endogenous from exogenous compounds and to detect, identify and quantify biomarker VOCs related to disease. Given that the concentrations of most volatile biomarkers in exhaled breath are low, often at parts-perbillion by volume, ppbv, or lower, this is a real challenge that the analytical instrumentation must meet and provide sufficiently accurate and precise to be useful clinically. If this can be performed directly both in real time and on-line avoiding sample collection that and provide immediate analytical results, then the most reliable breath analyses can be obtained. Such is the promise of SIFT-MS, as is shown below by sample data.

Unfortunately, so much breath analysis research is focused on efforts to simply identify volatile compounds of particular disease states by comparing the composition of breath from "healthy" subjects with breath from patients with particular disease states, paying too little attention to quantification that is essential to establish meaningful biomarkers. It is stating the obvious that it is of little value to inform a person that they have cholesterol in their blood without giving its concentration; the same should apply to breath biomarkers! Too often, most workers are apparently satisfied to reveal groups of breath biomarker compounds by statistical analysis of trace compound data derived from healthy/diseased groups of volunteers, rarely attempting to identify and quantify individual compounds. Such data are interesting and can be a guide to further research, but they are of little value to clinicians and are quickly archived. It is our contention that much more effort should be given to the identification and quantification of discrete breath biomarkers; in this we have had some success, as outlined later. It would be remiss not to mention that breath analysis is indeed used to effect in monitoring asthma via breath nitric oxide levels $[\underline{42}, \underline{43}]$ and in monitoring exhaled breath ${ }^{13} \mathrm{CO}_{2}$ following the ingestion of ${ }^{13} \mathrm{C}$-labelled compounds to pinpoint and track specific metabolic disorders and diseases $[\underline{44}, 45]$. Otherwise, the clinical return for the recent rapid increase in breath analysis research has so far been small, principally due to poorly directed research, some examples of which we describe in this article.

But it is not our intention in this "Perspective" to diminish the contribution of other users of SIFT-MS in their efforts to progress breath analysis and especially those involved in the search for biomarkers that could make an important contribution to clinical diagnosis and therapy monitoring. Rather, it is to steer the work towards a more rapid and clinically profitable approach to the desirable objectives that we all passionately desire. As mentioned above, we concentrate on breath analysis but not exclusively, because SIFT-MS is being profitably used in other areas, notably environmental (air pollution) monitoring and food 
flavour research that will briefly be referred to later and for which small instrumental development is also very desirable.

Breath sampling and analysis is essentially a simple, non-invasive and painless procedure that can be accomplished easily even by babies and frail patients. Sample collection by exhalation into bags followed by off-line analysis has been most commonly used, which inevitably provides a mixture of breath from the alveoli and the respiratory airways. Attempts have been made to selectively collect end-tidal breath (sometimes inaccurately termed alveolar breath) using exhaled carbon dioxide monitoring. This may be worthwhile ultimately, but at the expense of more complex, less direct sampling procedures that can compromise the breath sample due to condensation and cross contamination from additional tubing and circuitry. So we doubt that such efforts are really useful at the current stage of breath research, since they deflect from the much more challenging analytical issues involved in the search for real biomarkers. It is suggested by some that this sampling approach can minimise contamination of the mouth-exhaled breath sample by compounds generated in the oral cavity. This is pertinent, but it does not stand thorough examination since the exhaled breath still passes through the mouth. Yet it is true that mouth generation is not taken sufficiently serious in breath sampling and mostly is deliberately ignored. Surely, a better approach is to bypass the oral cavity by sampling breath exhaled via the nose? Of course, this presents an additional practical problem to breath sampling, and whilst not necessarily providing a true breath composition related to alveolar and airways origin only, it is a close approximation. We further address this situation below in relation to real time breath analysis that obviates breath sample collection and off-line analysis.

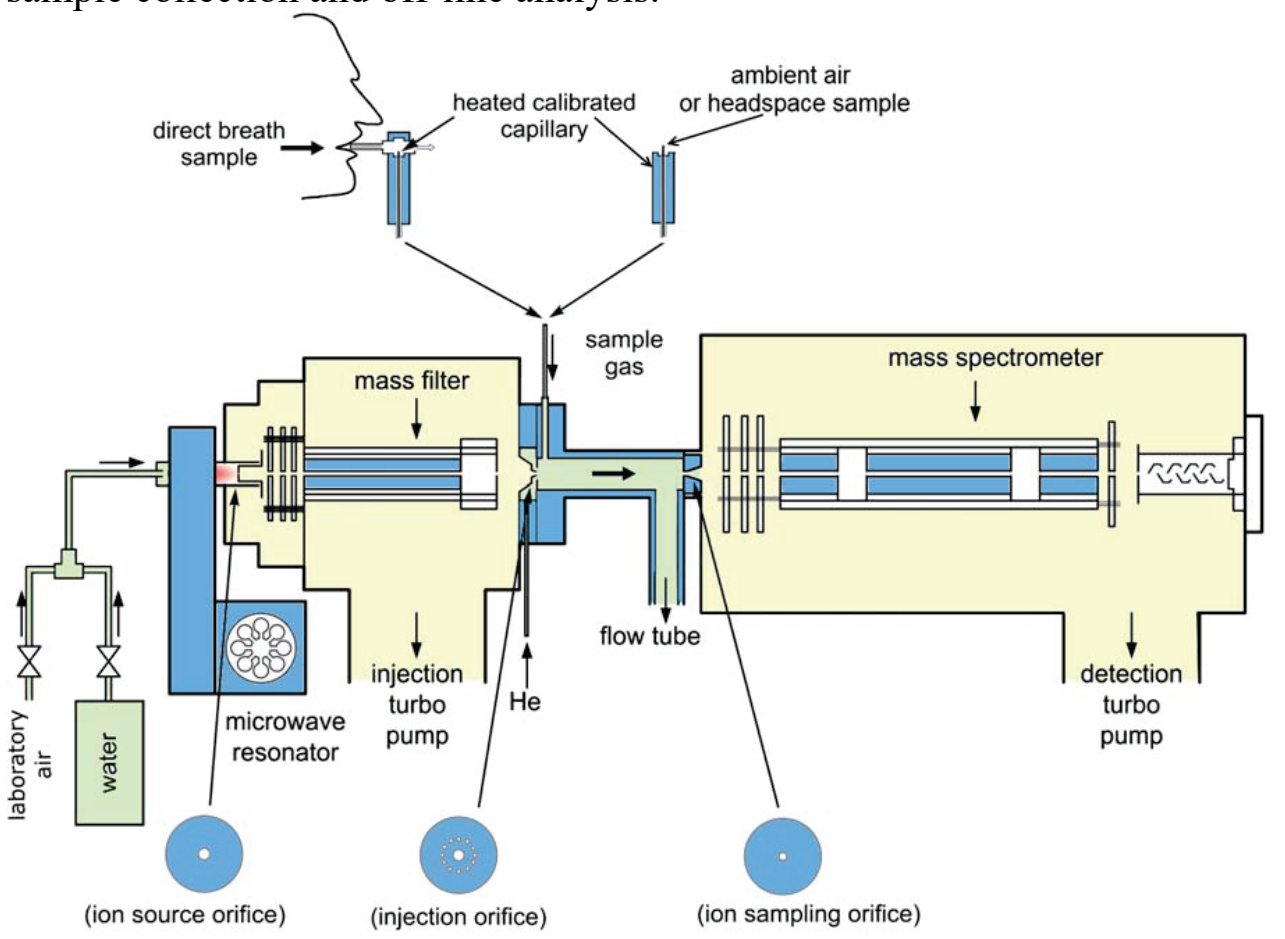

Fig. 1 A schematic diagram of the Profile 3 SIFT-MS instrument indicating the major functional parts. Decreasing ion currents pass sequentially through the three orifices indicated. Both direct breath sampling and sampling from ambient air or liquid headspace are depicted. Reproduced from [4] with permission from RSC. 
Breath analysis in real time by SIFT-MS is achieved by displacing the ambient air from the entrance to the heated calibrated capillary with directly-exhaled breath (see Figure 1) whilst running the detection mass spectrometer in either the FS or the MIM mode. The sampling entry lines are held at around $70 \mathrm{C}$ to inhibit surface adsorption of water vapour and condensable compounds. A disposable mouthpiece is used for mouth sampling of exhaled breath; inhalation through the mouth re-establishes ambient air at the capillary entrance for analysis. Several mouth exhalation/inhalation cycles are analysed for breath-by-breath consistency checks. Alternatively, breath sample collection into vessels for off-line analysis can be used, but water-soluble compounds such as ammonia can readily adsorb onto the vessel surfaces and thus distort the analysis. Bacterial filters must be used for on-line breath sampling from patients with infectious respiratory diseases. Typical data obtained for direct on-line breath sampling by SIFT-MS are shown in Figure 2 showing the well-defined exhalation time profiles and the derived concentrations of the several compounds that range from percentage levels (water vapour and carbon dioxide) to the ppbv levels of two trace metabolites (hydrogen cyanide and acetone) that carry the physiological and clinical data. $\mathrm{ppb}$

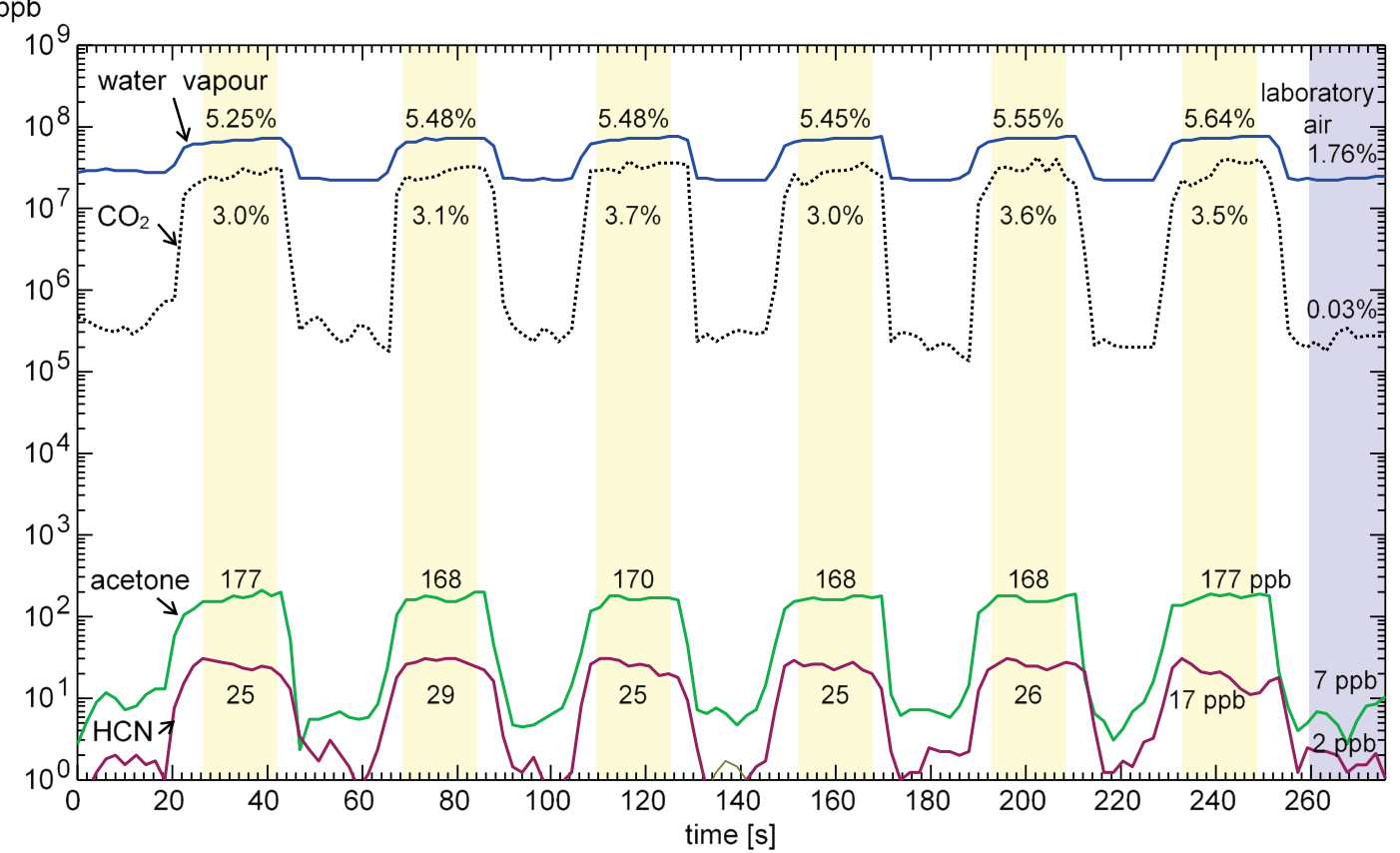

Fig. 2 Time concentration profiles for water vapour and $\mathrm{CO}_{2}$ (in \%) and acetone and $\mathrm{HCN}$ (in parts-per-billion by volume, ppb) for six sequential breath exhalations obtained using the MIM mode of the Profile 3 SIFT-MS instrument, indicating the remarkable breath-to-breath consistency in the derived concentrations of all four compounds. Also, indicated to the right, are the laboratory air concentrations of each compound. Reproduced from [4], with permission from RSC. 
What is so satisfying is that this sampling procedure can be easily modified to sample breath exhaled via the nose. Thus, a small vacuum pump is included in the SIFT-MS sampling line downstream in order to draw ambient air or exhaled breath across the entrance to the sampling capillary. The flow rate of air/breath must be adjusted to be much smaller than breath exhalation rates via the mouth or the nose to ensure that normal breath exhalations from the mouth and from the nose are not compromised. Then sampling of the exhaled breath from one nostril for about $10 \mathrm{~s}$ with both the second nostril and the mouth closed provides an on-line analysis of nose-exhaled breath. Closing both nostrils and sampling from the mouth indicates which compounds are predominantly produced in the oral cavity. Thus, analyses of specific selected compounds present in mouth-exhaled breath, nose-exhaled breath and mouth cavity air can be performed sequentially in real time in less than a minute. Such studies have provided very interesting data that show which compounds are predominantly produced in the oral cavity and those that are largely systemic. For example, ammonia and ethanol mostly originate in the oral cavity (influenced by oral hygiene) and acetone and isoprene are entirely systemic in origin. It is now apparent that much of the analyses carried out on mouth-exhaled breath are surely contaminated by orally-generated compounds and future studies must address this point. We have also demonstrated that collection of noseexhaled breath can be performed using a pump in the nose sampling line to inflate flexible bags, but it has to be appreciated that this can take a few minutes and so during the inhalation phase of breathing the sampled air will be diluted with laboratory air. This can be accounted for by normalising to the breath acetone level as measured directly in mouth exhalations and the laboratory air composition as measured during inhalations, according to the simple SIFTMS protocol outlined above. We have trialled this sampling procedure even by analysing the nose-exhaled breath of small babies with some success.

\section{Recent SIFT-MS studies: review and assessment}

The exploitation of SIFT-MS for trace gas analysis is growing in a number of fields and is limited currently mostly by the availability of affordable instrumentation. This undesirable situation needs to be rectified and we indicate the possible solution to this towards the end of this article. Even so, some hundreds of research papers have been published with SIFT-MS analyses as their focus and some reviews have been published that are a quick source of summary information [2-4, 25-29]. Unfortunately, some papers are uncritical and propagate some of the least desirable and unproductive approaches to SIFT-MS analyses. However, the results of much careful and thorough work have been reported notably by the groups of R. Dweik, C. Turner, B. Ross, G. Hanna in addition to our own contributions at Prague and Keele. But it must be said that the approaches of all these groups have their strengths and weaknesses; by critical appraisal, ways are variously suggested to better exploit SIFT-MS for trace gas analysis. We now briefly review and assess some of these studies that are largely directed towards disease detection, after which we address SIFT-MS through a SWOT analysis that, we hope, faithfully represents the current standing of SIFT-MS as a gas phase analytical method.

\section{- Ion chemistry: additional data}


To begin, it is appropriate to again recognise the requirement of a proper understanding of the ion chemistry that occurs in the SIFT-MS reactor to realise reliable analyses of humid air. If there is a general criticism of SIFT-MS work in most laboratories it is that not enough thought is given to this ion chemistry and proper quantification of trace compounds, which can be disastrous in medical studies. So it is desirable, even essential, to confirm the nature of assumed analytic ions of specific compounds formed under the specific conditions of the particular SIFT-MS instrument by the use of their pure compounds to confirmed the ion chemistry involved. Whilst this requires more work, it is more likely to produce reliable results. We also take the view that to be certain of compound identification, parallel GC-MS measurements should be carried out which, whilst not totally fool proof, can increase confidence in compound identification. It also makes good sense to attempt to trace the likely biochemistry forming breath biomarkers. Too little attention is given to these difficult aspects and the risk is that erroneous compound identification may take place.

Relevant ion chemistry studies continue to be reported, which can be of great help to SIFTMS practitioners. A good example is the recent work of Amelynck and his group [ $\underline{46}, 47]$ in which great efforts have been made to identify isomeric forms of terpenoid aldehydes, highlighting the fact that many biogenic trace compounds have several structural isomers that ideally should be characterised in analysis. Similar comments apply to isobaric compounds that are often difficult to separate in SIFT-MS, but this problem can be alleviated by using both $\mathrm{H}_{3} \mathrm{O}^{+}$and $\mathrm{NO}^{+}$, and perhaps $\mathrm{O}_{2}{ }^{+\bullet}$, reagent ions to analyse the sample, exemplified by a detailed study of the reactions of seven hexanol isomers [31]. Non-isomeric yet isobaric ions can also be separated with careful thinking, this being illustrated by the quantification of acetaldehyde, dimethyl sulphide and carbon dioxide involving isobaric analyte ions [48]. A recent, exciting study involves the study of the ion chemistry of malondialdehyde (MDA, see Figure 3) that has been proposed to reflect free oxygen-radical lipid peroxidation. This has allowed a kinetic library entry to be constructed for MDA in SIFT-MS which allows, for the first time, the detection of gaseous MDA as generated by in vitro cell cultures [18]. This study illustrates the effort that is often required to extend SIFT-MS analysis to biogenic compounds, which include many carboxylic acids for which the kinetics database entries have recently been constructed [32].

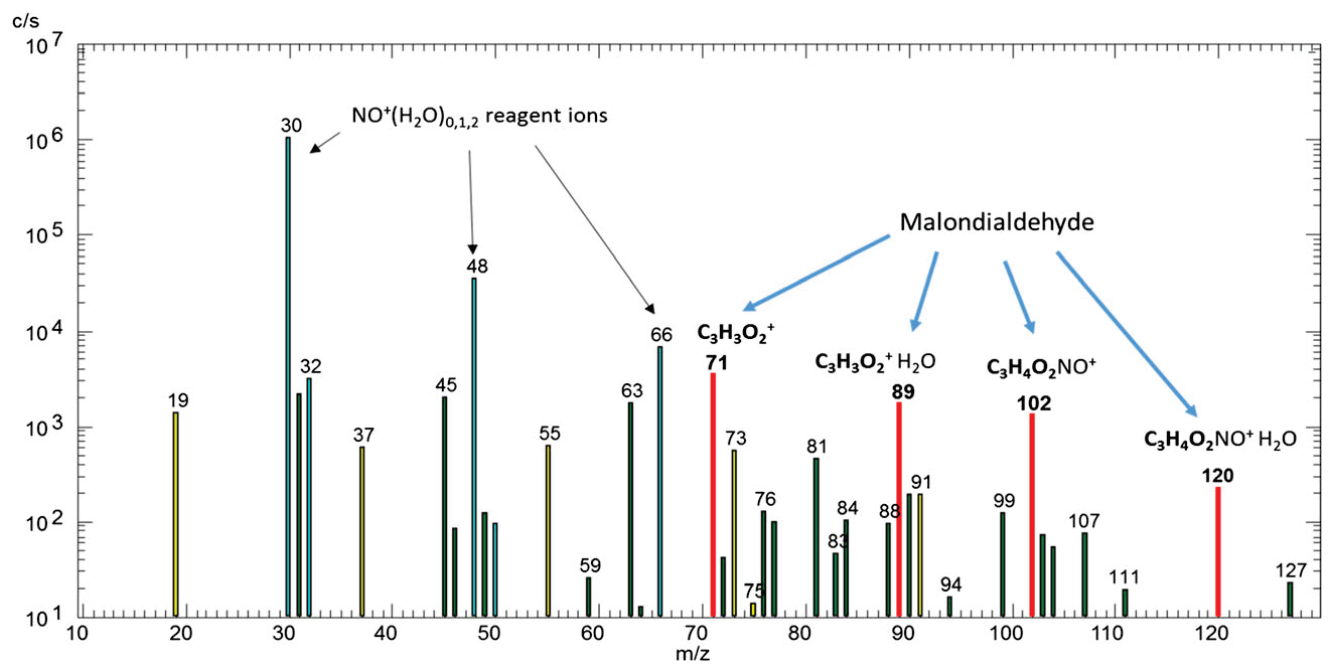

Fig 3. A full scan mass spectrum obtained when the headspace of freshly synthesised malondialdehyde (MW 72) water solution was analysed by SIFT-MS using $\mathrm{NO}^{+}$reagent ions showing four characteristic product ions at $\mathrm{m} / \mathrm{z}$ 71, 89, 102, 120; see text for further explanation. Reproduced from [18] with permission from Wiley. 
- Volatile metabolites in exhaled breath and released by cell cultures

Many SIFT-MS studies have been directed towards the detection in exhaled breath of volatile metabolites related to particular disease states. Notable contributions have been made by the aforementioned groups. Our approach to this potentially valuable disease diagnostic support has been to focus largely on the search for single compound breath biomarkers and with some success, as we review later. However, single volatile metabolite biomarkers of disease are considered by most workers in the field to be unlikely but why, given that there are numerous non-volatile blood single biomarkers routinely used for diagnosis? Hence, most breath researchers take a more pragmatic approach which is to identify groups of compounds that differentiate the composition of breath from patients with particular diseases from the breath of healthy controls.

Very active amongst the groups exploiting SIFT-MS is that of Raed Dweik at Cleveland, USA who, in this way, has made a valiant effort to demonstrate the value of breath analysis by several pointed studies, including the analysis of breath volatile metabolites as a noninvasive tool to diagnose chronic liver disease [49], non-alcoholic fatty liver disease in children [50], identifying unique "breathprint" in patients with inflammatory bowel disease (IBD) [51] and acute decompensated heart failure [52], "breathprints" in children with IBD [53] (see Figure 4) for obese children compared to lean children as controls [ $[54,55]$, children with juvenile idiopathic arthritis [56] and studies of breath volatile metabolites in patients with pouch disorders [ $\underline{57]}$.
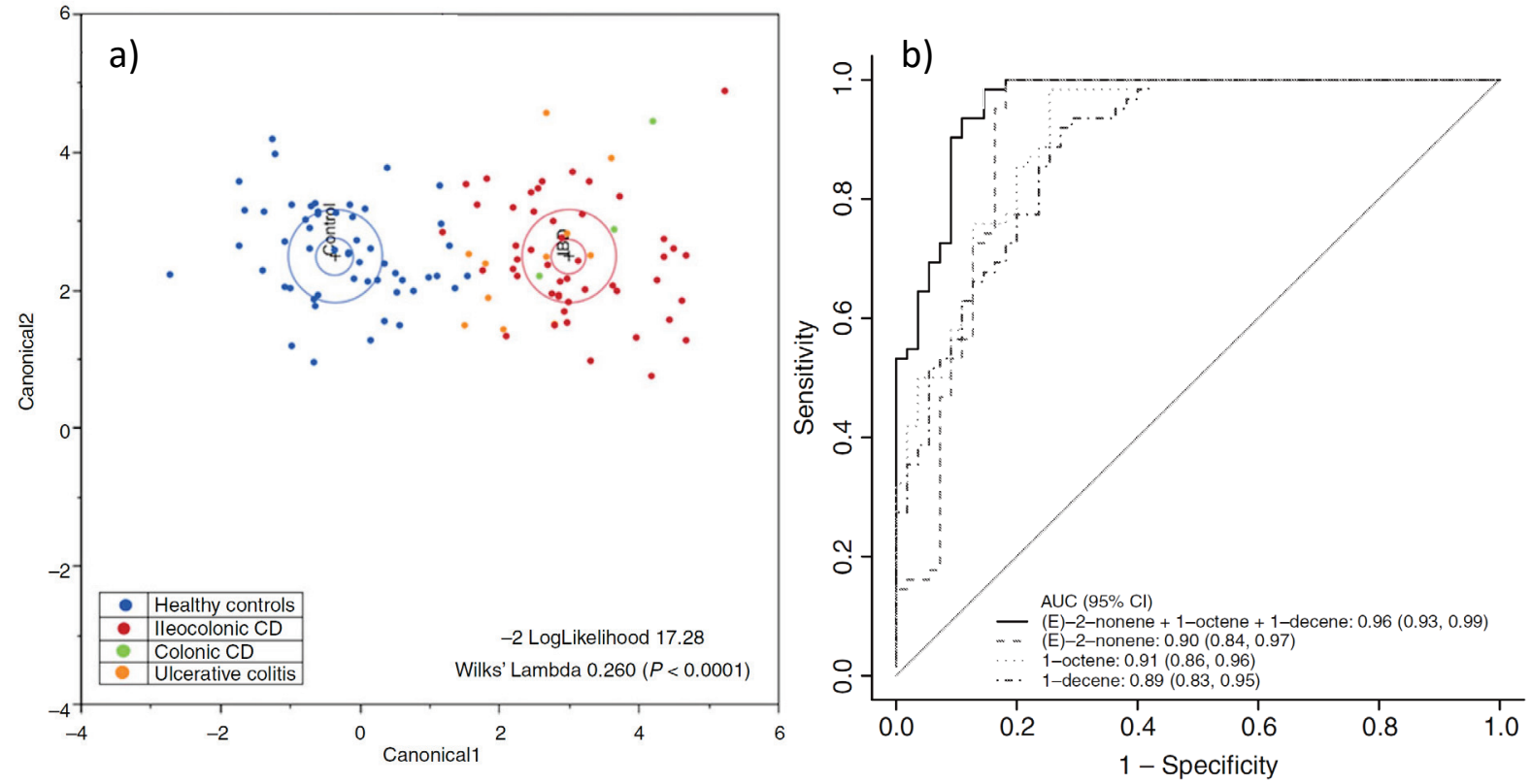

Fig. 4. Typical results of a SIFT-MS study aimed at discovery of combinations of VOCs discriminating between IBD patients and healthy controls [53]. (a) Visualisation of linear canonical discriminant analysis demonstrating that 21 pre-selected VOCs can classify patients with IBD or as healthy controls. (b) Receiver operating characteristic (ROC) curve analysis, demonstrating accuracy of predicting the presence of IBD by 1-octene, (E)2-nonene and 1-decene taken separately and in a linear model combination that gave the best area under the curve (AUC) of 0.96. Reproduced from [53] with permission from Wiley.

Differentiation between healthy and patient groups relies heavily on statistical analyses and often involves the measured concentrations of common and abundant breath compounds such as ammonia, acetone and isoprene that generally have a wide biological variability, even in the exhaled breath of healthy people, therefore having questionable validity. These analyses 
also often include rarer low concentration compounds that are sometimes difficult to definitely identify and quantify by SIFT-MS alone, especially when the ion chemistry complications in SIFT-MS analyses of humid breath described in the previous section are not fully appreciated. This is why we consider that parallel GC-MS (with pre-concentration techniques) are needed to support identification and quantification of low-level new compounds in exhaled breath. Whilst the derived results of this substantial body of research are thought-provoking, can they be more than just a prelude and a guide to further work and what is their value clinically at this early stage? Even the most carefully thought-out statistical analyses can lead to ambiguous and doubtful results, as we show later. A final comment relates to the use of the term "breathprint" for mass spectral patterns. If this is intended to be analogous with "fingerprint" it is misplaced, since in breath analysis use only a pattern is described and not the depth of the impression. It must be appreciated that for meaningful breath analysis in medicine the concentrations of the various biomarkers must be accurately measured.

Similar wide-ranging research is being carried out by the group of George Hanna at Imperial College London, UK, currently with a sharp focus on the detection and quantification of biomarkers in the breath (and urine) of oesophageal-gastric (OG) cancer patients, their surgical speciality. In a series of paper involving SIFT-MS analyses of exhaled breath and the headspace of gastric fluid and urine $[\underline{20}, \underline{22}, \underline{58-61]}$, they have searched for volatile biomarkers of OG cancer, anticipating that such could greatly help the early diagnosis and follow-up therapy after surgery. SPME/GC-MS and ATD/GC-MS have been added to their analytical armoury to firm up trace compound identification. [1]. Again, heavy reliance is placed on statistical analysis to differentiate component VOCs of patient samples and controls, including the use of optimisation of ROC curves to achieve diagnostic accuracy. Yet these ROC curves were constructed from the concentrations of several VOCs in combination, a questionable procedure that we consider later with respect to SIFT-MS data on breath analysis obtained in our Prague laboratory. An interesting and potentially important aspect of their investigations is the involvement of higher-order aldehydes (C3-C10) in tumour biogenesis, research that combines aldehyde detection and genetic manipulation of tumour cells concurrent with investigations of $\mathrm{C} 3-\mathrm{C} 10$ aldehydes in the exhaled breath of healthy subjects [62] This clever work holds real promise and signals a more imaginative approach to breath gas analysis by searching for the biochemical origins of the aldehydes. The pioneering work of this group in direct sampling of exhaled breath in the perioperative setting well demonstrates the real time analyses that are possible by SIFT-MS [7]. Recently, they have extended their research to the analysis of the exhaled breath of patients suffering from inflammatory bowel disease [63].

Claire Turner, formerly at the Silsoe Research Institute (SRI) and currently at the Open University, UK, is one of the original users of SIFT-MS for breath analysis [64-68]. The work of her research team has been varied and combines both the search for breath biomarkers using multivariate statistical methods and also the investigation of single biomarkers, notably acetone and its association with diabetes [69], which has realised some interesting results. The work of the group is notable in that it exploits several analytical methods and compares the data obtained from specific studies, exemplified by the use of GCMS, HPLC-MS and SIFT-MS in conjunction with multivariate classification for the diagnosis of Crohn's disease by urine analysis [70]. Recent work involves the development and use of portable breath sensor devices for monitoring diabetes and their quantitative validation using SIFT-MS [71], the analysis of the volatile faecal metabolome for screening of colorectal 

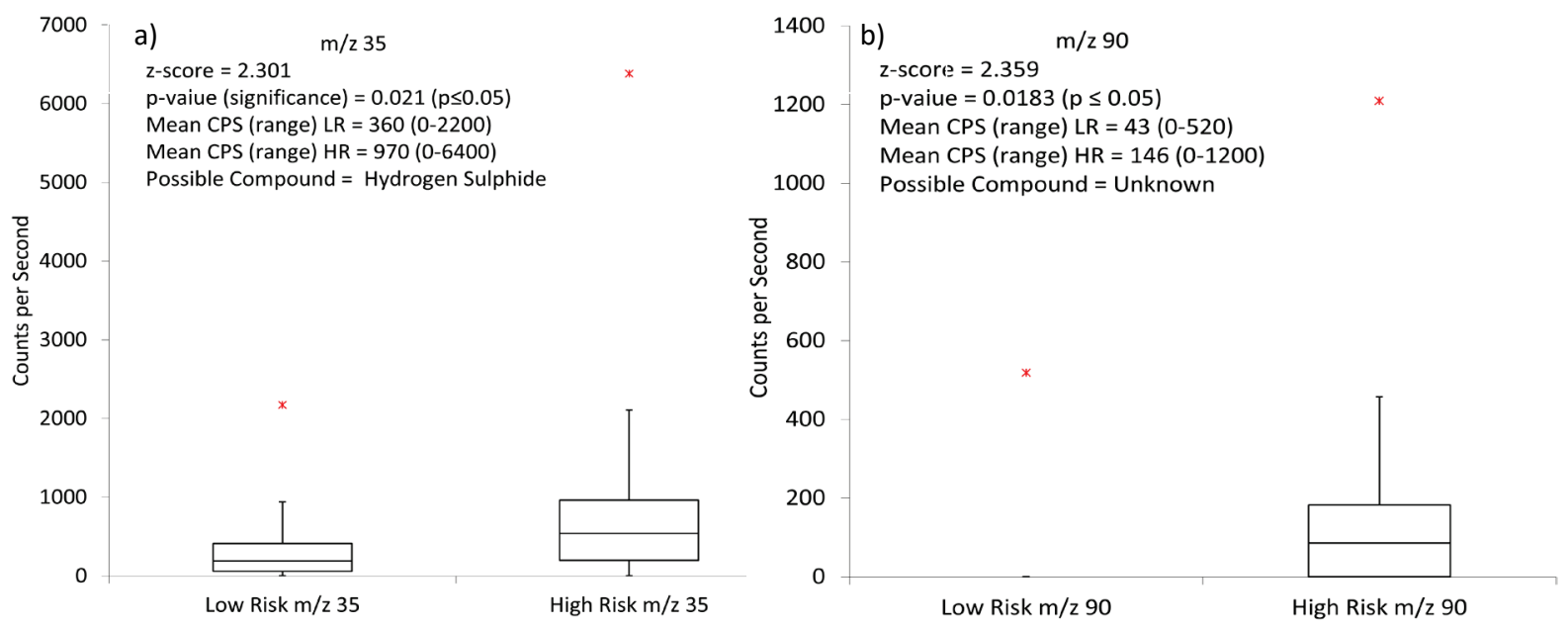

Fig. 5. Typical results of a SIFT-MS study aimed at discovery of single volatile metabolites in faecal metabolome as biomarkers of colorectal cancer [72]. (a) Results of SIFT-MS analyses of faecal headspace collected from low risk and high risk colorectal cancer groups obtained using $\mathrm{H}_{3} \mathrm{O}^{+}$reagent ions and the count rate of the analyte ion at $\mathrm{m} / \mathrm{z} 35$ (protonated hydrogen sulphide) (b) corresponding results for count rate at $\mathrm{m} / \mathrm{z}$ 90 (presumably a protonated nitrogen containing molecule). Reproduced from [72] under the Creative Commons Attribution.

441 VOCs faecal samples from rats fed control diets [73].

The work of the group of Brian Ross in Ontario, Canada, another pioneer of SIFT-MS, takes the eye being the first to combine sample concentration techniques (TD) with real time SIFTMS analysis to improve detection sensitivity $[\underline{10}, \underline{74}, \underline{75}$. The group has also recognised the importance of nose-exhaled breath analysis, as emphasized in the previous section, and combined TD and SIFT-MS to analyse nose-exhaled and mouth-exhaled breath showing that mouth-exhaled hydrogen sulphide [76], a known neuromodulator or cofactor, is largely generated in the oral cavity [77]. This work is truly exploiting the strength of SIFT-MS and shows one of its real advantages in breath analysis research. They also carried out the first meaningful studies of aldehydes as biomarkers of lipid peroxidation [78] and the release of these compounds from brain and liver cells following dietary supplementation with n-3 polyunsaturated fatty acids [79]. Their work has even reached towards food science [이 ] and plant and fungal science with the quantification of methanol both chemically and biologically generated from lignin [81].

SIFT-MS was conceived and developed at Keele by the authors subsequently with a major contribution to its development in Prague. During its continuous development as a research and commercial analytical tool it has been used in the manner original intended, which is as a real-time analytical technique that can uniquely contribute to the methods now available for trace gas analysis of ambient air and exhaled breath. Its development and much of the research work carried out by its exploitation up to 2011 by us and others are summarised in two major review papers $[\underline{3}, \underline{5}]$ and subsequent work is reviewed in more recent papers $[\underline{26}$, 28]. In relation to breath analysis, a major contribution has been the provision of concentration reference ranges of common breath metabolites, in close collaboration with the aforementioned Claire Turner [64]. These were constructed by direct on-line, real-time SIFT- 
MS analyses (obviating sample collection) of the exhaled breath of the healthy population. As far as disease detection is concerned, we have tried to focus on the detection and quantification of single breath biomarker compounds of specific diseases that, we believe, are most desirable, since they would be more readily utilized for clinical diagnosis and therapeutic monitoring. In this pursuit we have had some success, as summarised by the following:

- Serendipitous detection of gaseous hydrogen cyanide $(\mathrm{HCN})$ when analysing by SIFT-MS the volatile emissions from cultures of Pseudomonas aeruginosa (PA) bacteria derived from patients with cystic fibrosis (CF), initiated a decade of research at Keele and Prague on the presence of HCN in the headspace of in vitro cultures of different strains of PA and in the exhaled breath of patients with CF. This has established $\mathrm{HCN}$ as a reliable single and discrete biomarker of PA infection of the airways and lungs $[\underline{29}, \underline{82-86}]$.

- During this SIFT-MS PA/HCN research it was noticed that methyl thiocyanate was also emitted by PA cultures, its positive identification being confirmed by the analysis of the culture headspace with SPME/GC-MS following which SIFT-MS was used to quantify this VOC. [87, 88]. This was the first demonstration of the parallel use of SIFT-MS and GC-MS for trace gas analysis, a procedure that we now adopt routinely in the analysis of biogenic media and which we strongly encourage others to adopt.

- Detailed studies of exhaled breath of patients with Crohn's disease (CD) and ulcerative colitis (UC) have revealed elevated levels of n-pentane relative to healthy controls $[89,90]$. This required a detailed challenging study of the ion chemistry involved in n-pentane detection and quantification by SIFT-MS and which now allows this hydrocarbon to be detected in very humid breath, which can ultimately assist the non-invasive screening of inflammatory processes such as CD and UC.

- During a collaborative study with clinicians focused on the composition of exhaled breath from those suffering from gastroesophageal reflux disease (GERD), it was discovered that the only VOC elevated in their breath relative to controls was acetic acid. Again, both SIFT-MS and GC-MS were exploited for this study. This indicates that breath acetic acid may be a useful indicator of GERD and other conditions that result in a lowering of $\mathrm{pH}$ of the lining of the airways [21]. It is exciting to note that in a very recent study (as yet unpublished), acetic acid is observed to be greatly elevated in the exhaled breath of cystic fibrosis patients, which may have important implications to the treatment of this disease.

- Methane is present in the exhaled breath of all human beings and, along with hydrogen, when elevated it is a reflection of gut bacterial overload. We have developed SIFT-MS to quantify breath methane on-line in single breath exhalations using the slow reaction of $\mathrm{O}_{2}^{+*}$ reagent ions with methane [91]. Based on this work, breath methane concentration profiles have been constructed during exercise on an ergometer using the analogous PTR-MS real time analytical method [92], ostensibly providing a detector of gut bacterial overload.

- As the expected new innovators of SIFT-MS-type instrumentation, we must not remain complacent. Thus, thoughts are continuously directed to improving compound identification and analytical sensitivity. For the former, we consider the idea of introducing TOF-MS higher resolution analysis to differentiate isobaric ions, but this would not be a panacea [8] and would greatly increase the cost of instrumentation. To 
increase analytical sensitivity, new approaches to the thermal desorption of volatile compounds extracted from breath by sorbent tubes and its direct link to SIFT-MS are under development in Prague. Whilst acknowledging that the precious real-time direct analysis of exhaled breath and biogenic fluid headspace will be compromised, we believe that this will ultimately improve accuracy of quantification and simplify remote breath sample collection, transport and storage. Further to these developments, we have commenced research in the area of selected ion flow drift tube mass spectrometry, SIFDT-MS, which has some distinct advantages over SIFT-MS due to the inclusion of a low intensity drift field in the analytical reactor whilst retaining all the features of rapid analysis and immediate quantification [93, 94] (see Figure 6).

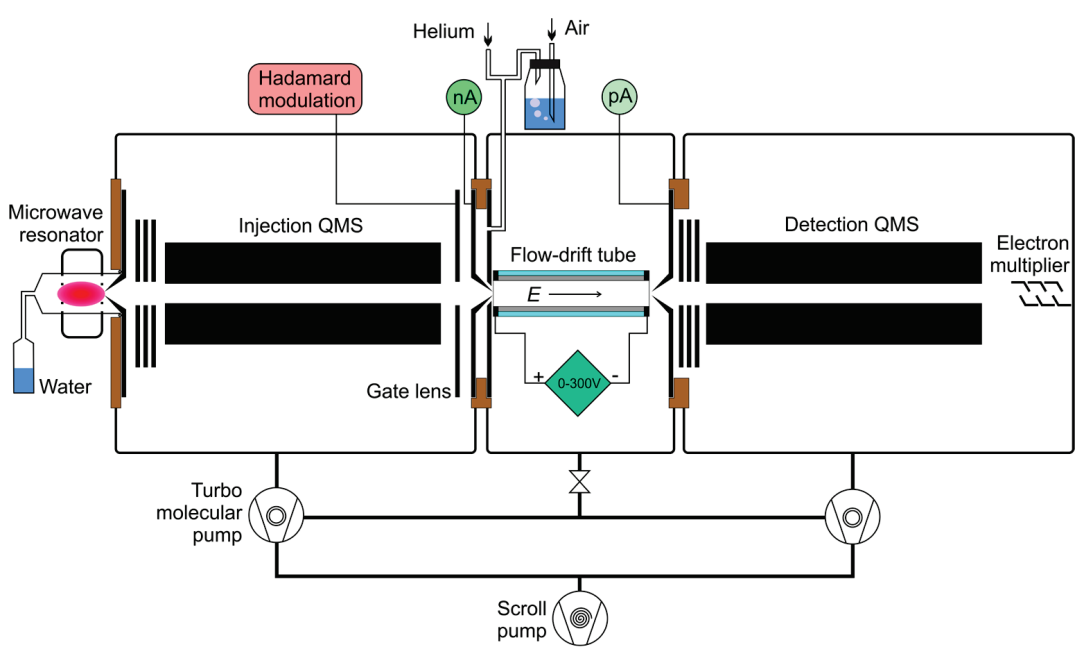

Fig. 6. Schematic drawing of the selected ion flow-drift tube, SIFDT, apparatus recently developed in Prague. In this representation the sample gas introduction setup is represented by a glass vial containing $10 \mathrm{~mL}$ of liquid water through which dry synthetic air or laboratory air is introduced but direct or bag samples of breath can be readily introduced. Note that the speed of the helium carrier gas is reduced by a restrictive aperture between the flow-drift tube compartment and a scroll pump in this case. Reproduced from [94] with permission from ACS.

The New Zealand groups (Malina Storer; Michael Epton) have made various contributions to breath research using the commercial SIFT-MS instruments Voice 100 and 200 (Syft Technologies, New Zealand), including both off-line and on-line measurement of exhaled HCN in relation to PA infection [95, 96], acetone in relation to diabetes [97] and critical illness [98]. They have also carried out breath testing in the workplace, particularly for acetonitrile exposure, the persuasive results of which are shown in Figure 7 [99]. A final development of note by Syft Technologies is the use of a SIFT-MS device Voice 200 loaded on a mobile van, which has been used for monitoring ambient air and exhaled breath for aromatic hydrocarbons to assess exposure to these compounds [100]. 


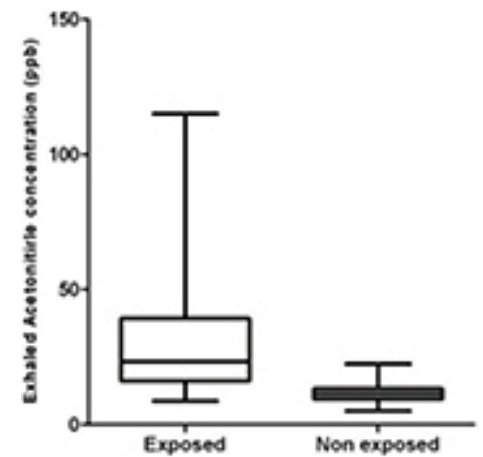

Fig. 7. Acetonitrile concentration measured by SIFT-MS in the exhaled breath of people who spent time working in a chemistry department laboratory where acetonitrile was used as a solvent. Reproduced with permission from IOP from [99].

\section{Some achievements using SIFT-MS in other areas}

The various areas in which SIFT-MS analysis is exploited are tabulated and reprised in a recent review [26] so they need not be discussed in detail here. However, in addition to breath research, worthy of mention is the significant work carried out in analysing VOCs from food flavours and processed food, largely by Sheryl Barringer in Ohio, USA [15, 101-104] and M Monica Flores in Valencia, Spain [105, 106]. Interesting work has also been done on the deodorization of breath following the ingestion of raw garlic by various food and food components, essentially to remove the odorous organosulphur compounds. It was observed that the enzymatic activity, of polyphenolic compounds and the acidity of specific foods may cause a reduction of these volatiles, but chlorophyll does not cause a deodorization effect. [107]

Studies of VOCs emitted by mammalian and bacterial cell cultures have been carried out in several laboratories following the early work [108-112], but it would neither do justice nor be appropriate to summarise these detailed programmes here; rather, the reader is referred to recent papers on the subject $[\underline{12}, \underline{108}, \underline{113-117]}$. But it is important to state that SIFT-MS real time analysis of the VOC emissions, including real time measurements during the evolution of bacterial cultures $[118, \underline{119}$ ], is ideal for these studies since it avoids sample collection and can be carried out over long (incubation) time periods.

\section{The strengths, weaknesses, opportunities and threats}

The strengths, weaknesses, opportunities and threats (SWOT) of and to SIFT-MS are worth analysing as we perceive them.

The strengths of SIFT-MS are articulated throughout this article. It is a powerful method for real-time breath analysis, ambient air analysis and the analysis of cell cultures headspace. It is an analytical method that uniquely can accurately and reliably measure concentrations of identified biomarkers of diseases in exhaled breath. To be useful clinically, such measurements must be objective and reproducible in all laboratories and clinics throughout the world, a requirement that analytical methods such as SPME/GC-MS can rarely fulfil without tedious and careful calibration. This is vital and important if reference ranges of 
breath metabolites are to be established in the same sense that are other crucial biomarkers such as blood pressure and cholesterol levels are accepted for health monitoring and control. The weaknesses of SIFT-MS relate to the limit of quantification, the practical lower limit being at about 1 ppbv in 1 second for real-time acceptably accurate analyses of trace metabolites in exhaled breath, and to the positive identification of unknown neutral trace gas analytes at characteristic analyte ions $\mathrm{m} / \mathrm{z}$ values above 100 and beyond. Lower limits of sensitivity have been reported for specific trace compounds in air but not yet realised for general use [11].

Thus, identification of isobaric and isomeric compounds is generally not possible, but the incorporation of TOF-MS could help. The operation of SIFT-MS instruments and data interpretation is not easy for technicians, scientists and health professionals that do not have knowledge of mass spectrometry and ion chemistry. To alleviate these weaknesses offers a real challenge to the future development of SIFT-MS-type devices.

Thus, there are obvious opportunities for instrumental development such as the improvement of analytical sensitivity and the reductions in size and cost, but importantly retaining the striking and unique features of real-time analyses and accurate quantification of trace compounds. These developments are now in train in our Prague laboratory which, when realised, will widen the exploitation of SIFT-MS in other areas of research and commerce such as, for example, in the surgeries of general practitioners where wide screening by realtime breath analysis could be carried out routinely and non-invasively for abnormal levels of disease-related breath metabolites.

The threats that are evident to the wider use of SIFT-MS in medicine largely revolve around the reluctance of clinicians to adopt breath analysis as an aid to clinical diagnoses and therapeutic monitoring even though it has great potential as a non-invasive diagnostic; this is de-motivating to research workers in this field. Their negative, often justified assessment is partly due to the failure of research workers in breath analysis to convincingly articulate their experimental work and to the premature reporting of inconsistent results that often defy previous work without explanation. There is even the tendency to ignore the essential requirements for biomarker identification and accurate quantification for the sake of expediency and the urge by institutional management authorities for more publications ("publish and be damned"). Additionally, the adoption of inadequate analytical methods that fail to identify genuine biomarkers and the excessive reliance on statistics to assess unreliable data is a major problem, as highlighted previously. Nevertheless, we believe that direct online SIFT-MS analysis offers the best hope for the acceptance of breath analysis into medical practice. It is perhaps significant that the wider growing use of SIFT-MS is in the less stringent areas of food monitoring and ambient trace gas detection as alluded to previously.

\section{Summary remarks}

What are the essential points that can be extracted from the above "Perspective"? They are that SIFT-MS is a unique and valuable addition to ambient trace gas analysis in that direct on-line analysis of volatile compounds in ambient air and very humid exhaled breath can be achieved. This offers a rapid, direct non-invasive analytical method to assist clinical diagnosis and therapeutic monitoring that is not offered by breath sample collection and offline GC-MS analysis. SIFT-MS analysis is relatively straightforward for the analysis of known trace compounds present at easily measurable concentrations. But when searching for and accurately analysing less common trace gases or breath metabolites, it must be used with aforethought with an eye on the underlying physics and chemistry in order to avoid serious 
errors that would damn this analytical method in the eyes of clinicians. An important point to stress is that the over reliance on statistical analysis when interpreting arrays of SIFT-MS (and GC-MS) data can often lead to misleading and even false results and deductions, which obviously is disastrous in medicine. Too little effort is given to the identification and accurate quantification of single breath metabolites that can be used as meaningful diagnostic biomarkers. Too much reliance is placed on patterns of several volatile metabolites that are collectively labelled as biomarkers, which will never to be exploited by clinicians. The strengths and weaknesses of SIFT-MS are made clear in this article, and the requirements for instrumental improvement are spelled out that, if achieved, will remove the threats to the extension and wider use of SIFT-MS in medicine and other areas.

\section{Future perspective}

A necessary development of SIFT-MS is the improvement of trace compound identification and analytical sensitivity. This is partly envisaged by commencing development of new method, SIFDT-MS, that includes a drift-tube reactor, and the inclusion of thermal desorption elements for analyses of samples collected on sorbent tubes.

It is anticipated that small hand-held instruments will be developed involving optical spectroscopy and solid state sensors to target individual biomarker molecules.

When further analyte ion resolution is needed to separate isobaric ions then the combination of SIFT-MS with GC-MS will be further exploited to guarantee more certain compound identification and accurate quantification of biomarkers that can be adopted in medicine.

Further efforts must be made to identify and quantify single breath biomarkers. When compound "profiling is the only way forward then better understanding of statistical methods for data interpretation need to be investigated. 
- As the need for the analysis of more complex trace compounds arises, the challenges to SIFT-MS analyses grow and more detailed ion chemistry investigations are required, especially associated with the involvement of adduct ion formation. To obtain more certain compound identification, it is strongly advised to combine GC-MS and SIFT-MS analyses, especially when isobaric and isomeric compounds are involved.

- The analysis of trace gases (metabolites) in humid exhaled breath presents a serious analytical challenge. SIFT-MS provides the best route to direct, noninvasive real time analysis of single breath exhalations.

- Guidance as to the best approach to breath analysis and examples of the data that can be obtained by SIFT-MS are given as a guideline to the required quality.

- Reliance on statistical analyses to distinguish "normal breath" from "diseased breath" should be diminished with greater focus on the identification of single biomarkers of disease that are much more likely to be adopted as useful biomarkers of disease.

- $\quad$ SIFT-MS has been exploited to monitor volatile organic compounds from food and food products in real time and those released by mammalian and bacterial cell cultures in vitro.

- Strengths, weaknesses, opportunities and threats (SWOT analysis) in SIFT-MS is given, an appreciation of which will further establish it as a valuable method in the panoply of analytical methods being developed for gas analysis. 


\section{Bibliography}

Papers of special note have been highlighted as:

* of interest

$* *$ of considerable interest

1. Smith D, Španěl P: Pitfalls in the analysis of volatile breath biomarkers; suggested solutions and SIFT-MS quantification of single metabolites. J. Breath Res. 9(2), 022001 (2015).

** A perspective article discussing the difficulties and the proper approach to SIFT-MS breath analysis

2. Smith D, Španěl P: Direct, rapid quantitative analyses of BVOCs using SIFT-MS and PTR-MS obviating sample collection. TrAC 30(7), 945-959 (2011).

3. Španěl P, Smith D: Progress in SIFT-MS: Breath analysis and other applications. Mass Spectrom. Rev. 30(2), 236-267 (2011).

** A detailed overview of the SIFT-MS technique and the areas of application.

4. Smith D, Španěl P: Ambient analysis of trace compounds in gaseous media by SIFTMS. Analyst 136(10), 2009-2032 (2011).

5. Smith D, Španěl P: Selected ion flow tube mass spectrometry (SIFT-MS) for on-line trace gas analysis. Mass Spectrom. Rev. 24(5), 661-700 (2005).

6. Španěl P, Dryahina K, Smith D: A general method for the calculation of absolute trace gas concentrations in air and breath from selected ion flow tube mass spectrometry data. Int. J. Mass Spectrom. 249, 230-239 (2006).

* A full description of the SIFT-MS analytical techniqe for absolute quantification.

7. Boshier PR, Cushnir JR, Mistry V et al.: On-line, real time monitoring of exhaled trace gases by SIFT-MS in the perioperative setting: a feasibility study. Analyst 136(16), 3233-3237 (2011).

8. Smith D, Španěl P, Herbig J, Beauchamp J: Mass spectrometry for real-time quantitative breath analysis. J. Breath Res. 8(2), 027101 (2014).

9. S S Spaněl P, Smith D: Advances in On-line Absolute Trace Gas Analysis by SIFT-MS. Curr. Anal. Chem. 9(4), 525-539 (2013).

10. Ross B: Sub-parts per billion detection of trace volatile chemicals in human breath using selected ion flow tube mass spectrometry. BMC Research Notes 1(1), 41 (2008).

11. Prince BJ, Milligan DB, Mcewan MJ: Application of selected ion flow tube mass spectrometry to real-time atmospheric monitoring. Rapid Commun. Mass Spectrom. 24(12), 1763-1769 (2010).

12. Chippendale TWE, Španěl P, Smith D, El Haj AJ: Counting cell number in situ by quantification of dimethyl sulphide in culture headspace. Analyst 139(19), 4903-4907 (2014).

13. Smith D, Bloor R, George C, Pysanenko A, Spanel P: Release of toxic ammonia and volatile organic compounds by heated cannabis and their relation to tetrahydrocannabinol content. Analytical Methods 7(10), 4104-4110 (2015).

14. Sovova K, Wiggins T, Markar SR, Hanna GB: Quantification of phenol in urine headspace using SIFT-MS and investigation of variability with respect to urinary concentration. Analytical Methods 7(12), 5134-5141 (2015).

15. $\mathrm{Xu} \mathrm{Y,} \mathrm{Barringer} \mathrm{S:} \mathrm{Comparison} \mathrm{of} \mathrm{tomatillo} \mathrm{and} \mathrm{tomato} \mathrm{volatile} \mathrm{compounds} \mathrm{in} \mathrm{the}$ headspace by selected ion flow tube mass spectrometry (SIFT-MS). J. Food Sci. 5, 268-273 (2013). 
16. Langford VS, Milligan DB, Prince BJ, Mcewan MJ: Application of selected ion flow tube mass spectrometry (SIFT-MS) to classifying coffee and parmesan cheese. Abstracts of Papers of the American Chemical Society 247, 1 (2014).

17. Heynderickx PM, Španěl P, Van Langenhove H: Quantification of octanol-water partition coefficients of several aldehydes in a bubble column using selected ion flow tube mass spectrometry. Fluid Phase Equilibria 367, 22-28 (2014).

18. Shestivska V, Antonowicz SS, Dryahina K, Kubišta J, Smith D, Španěl P: Direct detection and quantification of malondialdehyde vapour in humid air using selected ion flow tube mass spectrometry supported by gas chromatography/mass spectrometry. Rapid Commun. Mass Spectrom. 29(11), 1069-1079 (2015).

* First quantification of malondialdehyde in the gas phase.

19. Kumar S, Huang J, Abbassi-Ghadi N et al.: Mass Spectrometric Analysis of Exhaled Breath for the Identification of Volatile Organic Compound Biomarkers in Esophageal and Gastric Adenocarcinoma. Annals of surgery 262(6), 981-990 (2015).

20. Kumar S, Huang J, Abbassi-Ghadi N et al:: SIFT-MS analysis of exhaled breath for volatile organic profiling of oesophago-gastric cancer. Br. J. Surg. 101, 17-18 (2014).

21. Dryahina K, Pospisilova V, Sovova K et al:: Exhaled breath concentrations of acetic acid vapour in gastro-esophageal reflux disease. J. Breath Res. 8(3), 037109 (2014).

22. Kumar S, Huang JZ, Abbassi-Ghadi N, Španěl P, Smith D, Hanna GB: Selected Ion Flow Tube Mass Spectrometry Analysis of Exhaled Breath for Volatile Organic Compound Profiling of Esophago-Gastric Cancer. Anal. Chem. 85(12), 6121-6128 (2013).

23. Spanel P, Dryahina K, Vicherková P, Smith D: Increase of methanol in exhaled breath quantified by SIFT-MS following aspartame ingestion. J. Breath Res. In press, (2015).

24. Gilchrist F, Belcher J, Jones A et al.: Exhaled breath hydrogen cyanide as a marker of early Pseudomonas aeruginosa infection in children with cystic fibrosis. The Lancet Respiratory Medicine Submitted, (2015).

25. Smith D, Španěl P: The SIFT and FALP techniques; applications to ionic and electronic reactions studies and their evolution to the SIFT-MS and FA-MS analytical methods. Int. J. Mass Spectrom. 377(0), 467-478 (2015).

* Historical development of laboratory techniques to study kinetics into analytical methods.

26. Smith D, Spanel P: SIFT-MS and FA-MS methods for ambient gas phase analysis: developments and applications in the UK. Analyst 140(8), 2573-2591 (2015).

27. Davies SJ, Španěl P, Smith D: Breath analysis of ammonia, volatile organic compounds and deuterated water vapor in chronic kidney disease and during dialysis. Bioanalysis 6(6), 843-857 (2014).

28. Španěl P, Smith D: Account On the features, successes and challenges of selected ion flow tube mass spectrometry. Eur. J. Mass Spectrom. 19(4), 225-246 (2013).

29. Smith D, Španěl P, Gilchrist FJ, Lenney W: Hydrogen cyanide, a volatile biomarker of Pseudomonas aeruginosa infection. J. Breath Res. 7(4), 044001 (2013).

* A summary of a decade of research that establishes HCN as a biomarker of Pseudomonas aeruginosa infection.

30. Sovová K, Dryahina K, Španěl P: Selected ion flow tube (SIFT) studies of the reactions of $\mathrm{H}_{3} \mathrm{O}^{+}, \mathrm{NO}^{+}$and $\mathrm{O}_{2}{ }^{+\bullet}$ with six volatile phytogenic esters. Int. J. Mass Spectrom. 300(1), 31-38 (2011).

31. Smith D, Sovová K, Španěl P: A selected ion flow tube study of the reactions of $\mathrm{H}_{3} \mathrm{O}^{+}, \mathrm{NO}^{+}$and $\mathrm{O}_{2}{ }^{+}$with seven isomers of hexanol in support of SIFT-MS. Int. J. Mass Spectrom. 319, 25-30 (2012). 
32. Michalčíková RB, Španěl P: A selected ion flow tube study of the ion molecule association reactions of protonated $\left(\mathrm{MH}^{+}\right)$, nitrosonated $\left(\mathrm{MNO}^{+}\right)$and dehydroxidated $(\mathrm{M}-\mathrm{OH})^{(+)}$carboxylic acids (M) with $\mathrm{H}_{2} \mathrm{O}$. Int. J. Mass Spectrom. 368, 15-22 (2014).

33. Smith D, Chippendale TWE, Shpaněl P: Reactions of the selected ion flow tube mass spectrometry reagent ions $\mathrm{H}_{3} \mathrm{O}^{+}$and $\mathrm{NO}^{+}$with a series of volatile aldehydes of biogenic significance. Rapid Commun. Mass Spectrom. 28(17), 1917-1928 (2014).

34. Smith D, Pysanenko A, Španěl P: Ionic diffusion and mass discrimination effects in the new generation of short flow tube SIFT-MS instruments. Int. J. Mass Spectrom. 281(1-2), 15-23 (2009).

* A detailed description of the construction and analytical performance of the Profile 3 SIFT-MS instrument.

35. Španěl P, Smith D: Influence of water vapour on selected ion flow tube mass spectrometric analyses of trace gases in humid air and breath. Rapid Commun. Mass Spectrom. 14(20), 1898-1906 (2000).

36. Španěl P, Smith D: Influence of weakly bound adduct ions on breath trace gas analysis by selected ion flow tube mass spectrometry (SIFT-MS). Int. J. Mass Spectrom. 280(1-3), 128-135 (2009).

37. Pysanenko A, Španěl P, Smith D: A study of sulfur-containing compounds in mouthand nose-exhaled breath and in the oral cavity using selected ion flow tube mass spectrometry. J. Breath Res. 2(4), 046004 (2008).

* Significance of nose breath sampling to reduce the oral contribution to mouth exhaled metabolite concentrations.

38. Langford VS, Gray JDC, Maclagan RGaR, Milligan DB, Mcewan MJ: Real-time measurements of nitrosamines in air. Int. J. Mass Spectrom. 377, 490-495 (2015).

39. Zhan XF, Duan JN, Duan YX: Recent developments of proton-transfer reaction mass spectrometry (PTR-MS) and its applications in medical research. Mass Spectrom. Rev. 32(2), 143-165 (2013).

40. Joo E, Dewulf J, Demarcke $\mathrm{M}$ et al.: Quantification of interferences in PTR-MS measurements of monoterpene emissions from Fagus sylvatica L. using simultaneous TD-GC-MS measurements. Int. J. Mass Spectrom. 291(1-2), 90-95 (2010).

41. Španěl P, Smith D: On-line measurement of the absolute humidity of air, breath and liquid headspace samples by selected ion flow tube mass spectrometry. Rapid Commun. Mass Spectrom. 15(8), 563-569 (2001).

42. Heijkenskjold-Rentzhog C, Alving K, Kalm-Stephens P, Lundberg JO, Nordvall L, Malinovschi A: The fraction of NO in exhaled air and estimates of alveolar NO in adolescents with asthma: Methodological aspects. Pediatr. Pulmonol. 47(10), 941-949 (2012).

43. Sachs-Olsen C, Carlsen KCL, Mowinckel P et al.: Diagnostic value of exhaled nitric oxide in childhood asthma and allergy. Pediatr. Allergy Immunol. 21(1), E213-E221 (2010).

44. Opdam FL, Modak AS, Gelderblom H, Guchelaar HJ: Further characterization of a C13-dextromethorphan breath test for CYP2D6 phenotyping in breast cancer patients on tamoxifen therapy. J. Breath Res. 9(2), (2015).

45. Modak AS: An Update on C-13-Breath Tests: The Transition to Acceptability into Clinical Practice. Volatile Biomarkers: Non-Invasive Diagnosis in Physiology and Medicine, 245-262 (2013).

46. Amelynck C, Mees B, Schoon N, Bultinck P: FA-SIFT study of the reactions of $\mathrm{H}_{3} \mathrm{O}^{+}$ $\left(\mathrm{H}_{2} \mathrm{O}\right) \mathrm{n}(\mathrm{n}=0,1,2), \mathrm{NO}^{+}$and $\mathrm{O}_{2}{ }^{+}$with the terpenoid aldehydes citral, citronellal and myrtenal and their alcohol analogues. Int. J. Mass Spectrom. 379, 52-59 (2015). 
47. Amelynck C, Schoon N, Dhooghe F: SIFT Ion Chemistry Studies Underpinning the Measurement of Volatile Organic Compound Emissions by Vegetation. Curr. Anal. Chem. 9(4), 540-549 (2013).

48. Smith D, Chippendale TWE, Španěl P: Minimising the Effects of Isobaric Product Ions in SIFT-MS Quantification of Acetaldehyde, Dimethyl Sulphide and Carbon Dioxide. Curr. Anal. Chem. 9(4), 550-557 (2013).

49. Alsabbagh MEY, Chami AT, Gurshawn S et al.: Exhaled Breath Analysis Reveals New Biomarkers to Diagnose Advanced Fibrosis in Patients with Chronic Liver Disease. Hepatology 60, 417a-418a (2014).

50. Eng K, Alkhouri N, Cikach F et al:: Analysis of breath volatile organic compounds in children with chronic liver disease compared to healthy controls. J. Breath Res. 9(2), (2015).

51. Kurada S, Grove D, Alkhouri N et al:: A Specific Breath Metabolome Signature Identifies Patients With Inflammatory Bowel Diseases. Am. J. Gastroenterol. 110, S783-S783 (2015).

52. Samara MA, Tang WHW, Cikach F et al:: Single Exhaled Breath Metabolomic Analysis Identifies Unique Breathprint in Patients With Acute Decompensated Heart Failure. Journal of the American College of Cardiology 61(13), 1463-1464 (2013).

53. Patel N, Alkhouri N, Eng K et al.: Metabolomic analysis of breath volatile organic compounds reveals unique breathprints in children with inflammatory bowel disease: a pilot study. Aliment. Pharmacol. Ther. 40(5), 498-507 (2014).

* A very good example of SIFT-MS breath analysis revealing a group of volatile metabolites as biomarkers of a disease

54. Alkhouri N, Eng K, Cikach F et al.: Breathprints of childhood obesity: changes in volatile organic compounds in obese children compared with lean controls. Pediatric Obesity 10(1), 23-29 (2015).

55. Okwu V, Matloob A, Grove D et al:: Volatile Organic Compounds in the Exhaled Breath as Biomarkers of Nonalcoholic Steatohepatitis in Obese Children. Am. J. Gastroenterol. 109, S145-S145 (2014).

56. Zeft A, Costanzo D, Alkhouri N et al: Metabolomic Analysis of Breath Volatile Organic Compounds Reveals Unique Breathprints in Children With Juvenile Idiopathic Arthritis. Arthritis \& Rheumatology 66, S159-S159 (2014).

57. Kurada S, Grove D, Singh A et al.: The Breath Metabolome in Patients With Pouch Disorders. Am. J. Gastroenterol. 110, S797-S797 (2015).

58. Kumar S, Huang J, Hanna GB: SIFT-MS analysis of headspace vapour from gastric content for the diagnosis of gastro-oesophageal cancer. Br. J. Surg. 100, 4-4 (2013).

* A first example of SIFT-MS analysis of volaltile compounds emitted by liquid gastric content

59. Huang JZ, Kumar S, Boshier PR, Wakefield S, Cushnir JR, Hanna GB: Breath Analysis Using SIFT-MS to Assess Metabolic Status in Patients After Gastrooesophageal Cancer Surgery- a Pilot Study. Curr. Anal. Chem. 9(4), 584-592 (2013).

60. Huang JZ, Kumar S, Abbassi-Ghadi N, Spaněl P, Smith D, Hanna GB: Selected Ion Flow Tube Mass Spectrometry Analysis of Volatile Metabolites in Urine Headspace for the Profiling of Gastro-Esophageal Cancer. Anal. Chem. 85(6), 3409-3416 (2013).

61. Kumar S, Huang JZ, Cushnir JR, Španěl P, Smith D, Hanna GB: Selected Ion Flow Tube-MS Analysis of Headspace Vapor from Gastric Content for the Diagnosis of Gastro-Esophageal Cancer. Anal. Chem. 84(21), 9550-9557 (2012). 
62. Huang J, Kumar S, Hanna GB: Investigation of C3-C10 aldehydes in the exhaled breath of healthy subjects using selected ion flow tube-mass spectrometry (SIFT-MS). J Breath Res 8(3), 037104 (2014).

63. Hicks LC, Huang J, Kumar S et al:: Analysis of Exhaled Breath Volatile Organic Compounds in Inflammatory Bowel Disease: A Pilot Study. J. Crohns Colitis 9(9), 731-737 (2015).

64. Smith D, Turner C, Španěl P: Volatile metabolites in the exhaled breath of healthy volunteers: their levels and distributions. J. Breath Res. 1(1), 014004 (2007).

* A review of the concentrations of several metabolites in the exhaled breath of the normal population.

65. Turner C, Španěl P, Smith D: A longitudinal study of ammonia, acetone and propanol in the exhaled breath of 30 subjects using selected ion flow tube mass spectrometry, SIFT-MS. Physiol. Meas. 27(4), 321-337 (2006).

66. Turner C, Španěl P, Smith D: A longitudinal study of breath isoprene in healthy volunteers using selected ion flow tube mass spectrometry (SIFT-MS). Physiol. Meas. 27(1), 13-22 (2006).

67. Turner C, Španěl P, Smith D: A longitudinal study of ethanol and acetaldehyde in the exhaled breath of healthy volunteers using selected-ion flow-tube mass spectrometry. Rapid Commun. Mass Spectrom. 20(1), 61-68 (2006).

68. Turner C, Španěl P, Smith D: A longitudinal study of methanol in the exhaled breath of 30 healthy volunteers using selected ion flow tube mass spectrometry, SIFT-MS. Physiol. Meas. 27(7), 637-648 (2006).

69. Turner C, Walton C, Hoashi S, Evans M: Breath acetone concentration decreases with blood glucose concentration in type I diabetes mellitus patients during hypoglycaemic clamps. J. Breath Res. 3(4), (2009).

70. Cauchi M, Fowler DP, Walton C et al:: Comparison of GC-MS, HPLC-MS and SIFTMS in conjunction with multivariate classification for the diagnosis of Crohn's disease in urine. Analytical Methods 7(19), 8379-8385 (2015).

71. Walton C, Patel M, Pitts D et al.: The use of a portable breath analysis device in monitoring type 1 diabetes patients in a hypoglycaemic clamp: validation with SIFTMS data. J. Breath Res. 8(3), (2014).

72. Batty CA, Cauchi M, Lourenco C, Hunter JO, Turner C: Use of the Analysis of the Volatile Faecal Metabolome in Screening for Colorectal Cancer. Plos One 10(6), (2015).

* An example of a SIFT-MS study revealing the concetrations of single compounds as breath biomarkers.

73. Hopes K, Cauchi M, Walton C, Macqueen H, Wassif W, Turner C: A novel method for the analysis of clinical biomarkers to investigate the effect of diet on health in a rat model. Analyst 140(9), 3028-3038 (2015).

74. Hryniuk A, Ross BM: Detection of acetone and isoprene in human breath using a combination of thermal desorption and selected ion flow tube mass spectrometry. Int. J. Mass Spectrom. 285(1-2), 26-30 (2009).

75. Ross BM, Vermeulen N: The combined use of thermal desorption and selected ion flow tube mass spectrometry for the quantification of xylene and toluene in air. Rapid Commun. Mass Spectrom. 21(22), 3608-3612 (2007).

* The first use of ATD and SIFT-MS in combination for trace gas analysis.

76. Wondimu T, Wang R, Ross BM: A Comparison of Moisture Removing Strategies for Breath Samples Spiked with Trace Concentrations of Hydrogen Sulphide. Curr. Anal. Chem. 9(2), 312-318 (2013). 
77. Wondimu T, Wang R, Ross B: Hydrogen sulphide in human nasal air quantified using thermal desorption and selected ion flow tube mass spectrometry. J. Breath Res. 8(3), 8 (2014).

78. Ross BM, Puukila S, Malik I et al.: The Use of SIFT-MS to Investigate Headspace Aldehydes as Markers of Lipid Peroxidation. Curr. Anal. Chem. 9(4), 600-613 (2013).

79. Ross BM, Babay S, Malik I: Brain and Liver Headspace Aldehyde Concentration Following Dietary Supplementation with n-3 Polyunsaturated Fatty Acids. Lipids 50(11), 1123-1131 (2015).

80. Amadei G, Ross BM: Quantification of character-impacting compounds in Ocimum basilicum and 'Pesto alla Genovese' with selected ion flow tube mass spectrometry. Rapid Commun. Mass Spectrom. 26(3), 219-225 (2012).

81. Malek L, Gibson A, Dekker RFH, Ross BM: Sift-Ms Analysis of Methanol Chemically and Biologically Generated from Lignin. J-for-Journal of Science \& Technology for Forest Products and Processes 4(2), 6-10 (2014).

82. Gilchrist FJ, Bright-Thomas RJ, Jones AM et al.: Hydrogen cyanide concentrations in the breath of adult cystic fibrosis patients with and without Pseudomonas aeruginosa infection. J. Breath Res. 7(2), 026010 (2013).

83. Gilchrist FJ, Sims H, Alcock A et al.: Quantification of hydrogen cyanide and 2aminoacetophenone in the headspace of Pseudomonas aeruginosa cultured under biofilm and planktonic conditions. Analytical Methods 4(11), 3661-3665 (2012).

84. Gilchrist FJ, Razavi C, Webb AK et al.: An investigation of suitable bag materials for the collection and storage of breath samples containing hydrogen cyanide. J. Breath Res. 6(3), 036004 (2012).

85. Gilchrist FJ, Alcock A, Belcher $\mathrm{J}$ et al.: Variation in hydrogen cyanide production between different strains of Pseudomonas aeruginosa. European Respiratory Journal 38(2), 409-414 (2011).

86. Enderby B, Smith D, Carroll W, Lenney W: Hydrogen Cyanide as a Biomarker for Pseudomonas Aeruginosa in the Breath of Children With Cystic Fibrosis. Pediatr. Pulmonol. 44(2), 142-147 (2009).

87. Shestivska V, Španěl P, Dryahina K et al.: Variability in the concentrations of volatile metabolites emitted by genotypically different strains of Pseudomonas aeruginosa. $J$. Appl. Microbiol. 113(3), 701-713 (2012).

* SIFT-MS and GC-MS analysies of volatile metabolites from 36 genetically different strains

88. Shestivska V, Nemec A, Drevinek P, Sovová K, Dryahina K, Španěl P: Quantification of methyl thiocyanate in the headspace of Pseudomonas aeruginosa cultures and in the breath of cystic fibrosis patients by selected ion flow tube mass spectrometry. Rapid Commun. Mass Spectrom. 25(17), 2459-2467 (2011).

89. Dryahina K, Španěl P, Pospisilova V et al.: Quantification of pentane in exhaled breath, a potential biomarker of bowel disease, using selected ion flow tube mass spectrometry. Rapid Commun. Mass Spectrom. 27(17), 1983-1992 (2013).

90. Hrdlicka L, Dryahina K, Španěl P et al.: Noninvasive Quantification of Volatile Metabolites in Breath: A Potential Indicator of Inflammatory Bowel Diseases Activity. Gastroenterology 142(5), S784-S784 (2012).

91. Dryahina K, Smith D, Španěl P: Quantification of methane in humid air and exhaled breath using selected ion flow tube mass spectrometry. Rapid Commun. Mass Spectrom. 24(9), 1296-1304 (2010).

92. Szabo A, Ruzsanyi V, Unterkofler K et al: : Exhaled methane concentration profiles during exercise on an ergometer. J. Breath Res. 9(1), 016009 (2015). 
93. Spesyvyi A, Španěl P: Determination of residence times of ions in a resistive glass selected ion flow-drift tube using the Hadamard transformation. Rapid Commun. Mass Spectrom. 29(17), 1563-1570 (2015).

94. Spesyvyi A, Smith D, Spanel P: Selected Ion Flow-Drift Tube Mass Spectrometry: Quantification of Volatile Compounds in Air and Breath. Anal. Chem. 87(24), 1215112160 (2015).

* The first description of the SIFDT-MS analytical method

95. Dummer J, Storer M, Sturney S et al:: Quantification of hydrogen cyanide $(\mathrm{HCN})$ in breath using selected ion flow tube mass spectrometry-HCN is not a biomarker of Pseudomonas in chronic suppurative lung disease. J. Breath Res. 7(1), 017105 (2013).

96. Chambers ST, Scott-Thomas A, Epton M: Developments in novel breath tests for bacterial and fungal pulmonary infection. Current Opinion in Pulmonary Medicine 18(3), 228-232 (2012).

97. Storer M, Dummer J, Lunt H et al.: Measurement of breath acetone concentrations by selected ion flow tube mass spectrometry in type 2 Diabetes. J. Breath Res. 5(4), 046011 (2011).

98. Sturney SC, Storer MK, Shaw GM, Shaw DE, Epton MJ: Off-line breath acetone analysis in critical illness. J. Breath Res. 7(3), 9 (2013).

99. Storer M, Curry K, Squire M, Kingham S, Epton M: Breath testing and personal exposure-SIFT-MS detection of breath acetonitrile for exposure monitoring. J. Breath Res. 9(3), (2015).

* Demonstrating the use of SIFT-MS analysis in exposure studies

100. Storer M, Salmond J, Dirks KN, Kingham S, Epton M: Mobile selected ion flow tube mass spectrometry (SIFT-MS) devices and their use for pollution exposure monitoring in breath and ambient air-pilot study. J. Breath Res. 8(3), 037106 (2014).

101. Sumonsiri N, A Barringer S: Application of SIFT-MS in Monitoring Volatile Compounds in Fruits and Vegetables. Curr. Anal. Chem. 9(4), 631-641 (2013).

102. Akpolat H, Barringer SA: The Effect of $\mathrm{pH}$ and Temperature on Cabbage Volatiles During Storage. Journal of Food Science 80(8), S1878-S1884 (2015).

103. Smith AL, Perry JJ, Marshall JA, Yousef AE, Barringer SA: Oven, Microwave, and Combination Roasting of Peanuts: Comparison of Inactivation of Salmonella Surrogate Enterococcus faecium, Color, Volatiles, Flavor, and Lipid Oxidation. Journal of Food Science 79(8), S1584-S1594 (2014).

104. Smith AL, Barringer SA: Color and Volatile Analysis of Peanuts Roasted Using Oven and Microwave Technologies. Journal of Food Science 79(10), C1895-C1906 (2014).

105. Flores M, Olivares A, Dryahina K, Španěl P: Real Time Detection of Aroma Compounds in Meat and Meat Products by SIFT-MS and Comparison to Conventional Techniques (SPME-GC-MS). Curr. Anal. Chem. 9(4), 622-630 (2013).

106. Olivares A, Dryahina K, Španěl P, Flores M: Rapid detection of lipid oxidation in beef muscle packed under modified atmosphere by measuring volatile organic compounds using SIFT-MS. Food Chem. 135(3), 1801-1808 (2012).

107. Munch R, Barringer SA: Deodorization of Garlic Breath Volatiles by Food and Food Components. Journal of Food Science 79(4), C526-C533 (2014).

* Demonstrating the influence of odorous foods on breath trace compounds.

108. Smith D, Wang TS, Sule-Suso J, Španěl P, El Haj A: Quantification of acetaldehyde released by lung cancer cells in vitro using selected ion flow tube mass spectrometry. Rapid Commun. Mass Spectrom. 17(8), 845-850 (2003). 
109. Wang TS, Smith D, Španěl P: Selected ion flow tube, SIFT, studies of the reactions of $\mathrm{H}_{3} \mathrm{O}^{+}, \mathrm{NO}^{+}$and $\mathrm{O}_{2}{ }^{+}$with compounds released by Pseudomonas and related bacteria. Int. J. Mass Spectrom. 233(1-3), 245-251 (2004).

110. Allardyce RA, Hill AL, Murdoch DR: The rapid evaluation of bacterial growth and antibiotic susceptibility in blood cultures by selected ion flow tube mass spectrometry. Diagn. Microbiol. Infect. Dis. 55(4), 255-261 (2006).

111. Allardyce RA, Langford VS, Hill AL, Murdoch DR: Detection of volatile metabolites produced by bacterial growth in blood culture media by selected ion flow tube mass spectrometry (SIFT-MS). J. Microbiol. Methods 65(2), 361-365 (2006).

112. Scotter JM, Allardyce RA, Langford V, Hill A, Murdoch DR: The rapid evaluation of bacterial growth in blood cultures by selected ion flow tube-mass spectrometry (SIFTMS) and comparison with the BacT/ALERT automated blood culture system. $J$. Microbiol. Methods 65(3), 628-631 (2006).

113. Chingin K, Liang JC, Chen HW: Direct analysis of in vitro grown microorganisms and mammalian cells by ambient mass spectrometry. Rsc $A d v$ 4(11), 5768-5781 (2014).

114. Španěl P, Smith D: Recent SIFT-MS Studies of Volatile Compounds in Physiology, Medicine and Cell Biology. In: Volatile Biomarkers: Non-Invasive Diagnosis in Physiology and Medicine, Amann A,Smith D (Ed.^(Eds). Elsevier, Boston 49-76 (2013).

115. Rutter AV, Chippendale TWE, Yang Y, Španěl P, Smith D, Sule-Suso J: Quantification by SIFT-MS of acetaldehyde released by lung cells in a 3D model. Analyst 138(1), 91-95 (2013).

116. Chippendale TWE, Hu B, El Haj AJ, Smith D: A study of enzymatic activity in cell cultures via the analysis of volatile biomarkers. Analyst 137(20), 4677-4685 (2012).

117. Sule-Suso J, Pysanenko A, Španěl P, Smith D: Quantification of acetaldehyde and carbon dioxide in the headspace of malignant and non-malignant lung cells in vitro by SIFT-MS. Analyst 134(12), 2419-2425 (2009).

118. Chippendale TWE, Španěl P, Smith D: Time-resolved selected ion flow tube mass spectrometric quantification of the volatile compounds generated by E. coli JM109 cultured in two different media. Rapid Commun. Mass Spectrom. 25(15), 2163-2172 (2011).

* The first real time analysis of dynamic changes in volatile emissions from bacterial cultures.

119. Sovova K, Cepl J, Markos A, Španěl P: Real time monitoring of population dynamics in concurrent bacterial growth using SIFT-MS quantification of volatile metabolites. Analyst 138(17), 4795-4801 (2013). 